\title{
BMJ Open Survey of the capacity for essential surgery and anaesthesia services in Papua New Guinea
}

\author{
Janet Martin, ${ }^{1,2}$ Goa Tau, ${ }^{3}$ Meena Nathan Cherian, ${ }^{4}$ Jennifer Vergel de Dios, ${ }^{1}$ \\ David Mills, ${ }^{5}$ Jane Fitzpatrick, ${ }^{6}$ William Adu-Krow, ${ }^{7}$ Davy Cheng ${ }^{1}$
}

To cite: Martin J, Tau G, Cherian MN, et al. Survey of the capacity for essential surgery and anaesthesia services in Papua New Guinea. BMJ Open 2015;5: e009841. doi:10.1136/ bmjopen-2015-009841

- Prepublication history for this paper is available online. To view these files please visit the journal online (http://dx.doi.org/10.1136/ bmjopen-2015-009841).

Received 2 September 2015 Revised 10 November 2015 Accepted 17 November 2015

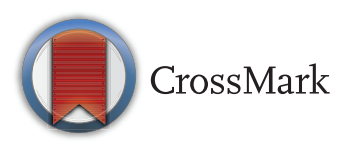

For numbered affiliations see end of article.

Correspondence to Dr Davy Cheng;

Davy.Cheng@Ihsc.on.ca

\section{ABSTRACT}

Objective: To assess capacity to provide essential surgical services including emergency, obstetric and anaesthesia care in Papua New Guinea (PNG) in order to support planning for relevant post-2015 sustainable development goals for PNG.

Design: Cross-sectional survey.

Setting: Hospitals and health facilities in PNG.

Participants: 21 facilities including 3 national/ provincial hospitals, 11 district/rural hospitals, and 7 health centres.

Outcome measures: The WHO Situational Analysis Tool to Assess Emergency and Essential Surgical Care (WHO-SAT) was used to measure each participating facility's capacity to deliver essential surgery and anaesthesia services, including 108 items related to relevant infrastructure, human resources, interventions and equipment.

Results: While major surgical procedures were provided at each hospital, fewer than $30 \%$ had uninterrupted access to oxygen, and $57 \%$ had uninterrupted access to resuscitation bag and mask. Most hospitals reported capacity to provide general anaesthesia, though few hospitals reported having at least one certified surgeon, obstetrician and anaesthesiologist. Access to anaesthetic machines, pulse oximetry and blood bank was severely limited. Many non-hospital health centres providing basic surgical procedures, but almost none had uninterrupted access to electricity, running water, oxygen and basic supplies for resuscitation, airway management and obstetric services.

Conclusions: Capacity for essential surgery and anaesthesia services is severely limited in PNG due to shortfalls in physical infrastructure, human resources, and basic equipment and supplies. Achieving post2015 sustainable development goals, including universal healthcare, will require significant investment in surgery and anaesthesia capacity in PNG.

\section{INTRODUCTION}

The Independent State of Papua New Guinea (PNG) is a nation of islands in the southwestern Pacific Ocean with a culturally

\section{Strengths and limitations of this study}

- Provides a first-time cross-sectional measure of capacity to provide essential surgical, emergency and anaesthesia services, with a broad representation of regions across Papua New Guinea.

- Detailed measures of infrastructure, human resources, interventions, supplies and equipment reveals significant gaps for some of the most basic needs (personnel, oxygen, other basic resuscitative equipment), and provides evidencebased priorities for planning for achieving post-2015 sustainable development goals, including essential surgery services as a component of universal healthcare.

- Does not provide measures from every hospital and health centre in the country.

- In some cases, reported data rely on subjective reports or estimates from hospital administrators, without opportunity for objective verification.

and linguistically diverse population that is expected to exceed 13 million by $2030 .{ }^{1-4}$ Over $80 \%$ of the population lives in rural settings across 300 islands, with over 800 official languages..$^{5-8}$ Life expectancy at birth is 62.8 years. ${ }^{2}{ }^{3}$ In the PNG Population Health Survey, maternal mortality rate was reported to be 733 per 100000 live births (2006), ${ }^{4}$ while other estimates indicate the rate may be closer to 230 per 100000 live births in recent years. ${ }^{2}$ The under-5 mortality rate has improved from 74.7 to 61 per 1000 live births in the recent 5 years. ${ }^{2}$ The gross national income per capita is $\$ 2010$, which meets the definition of low-middle income country (LMIC)..$^{5}$ On the United Nation's Human Development Index (HDI) ranking, PNG is 157 out of 187 countries worldwide, ${ }^{6}$ with a slow improvement over the past 30 years (figure 1).

A global meta-analysis demonstrated clear reductions in perioperative mortality over the past 50 years, despite increasing complexity 


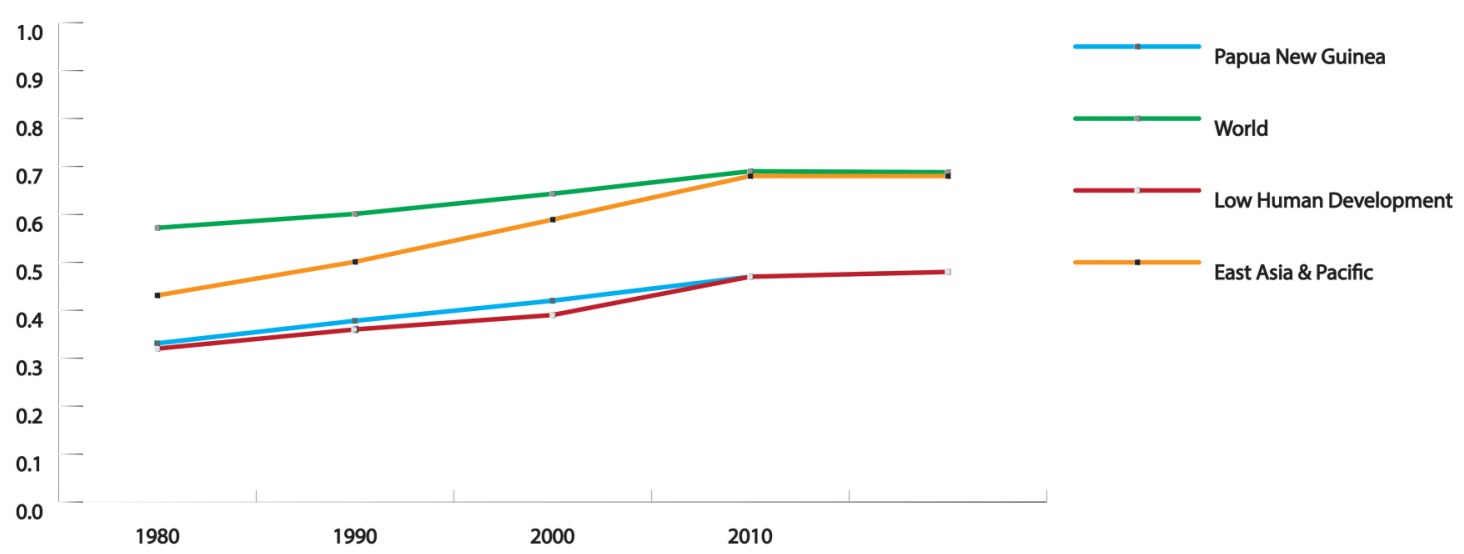

Figure 1 Human Development Index (HDI): trends for Papua New Guinea relative to other low HDI countries, and relative to the East Asia and the Pacific: $1980-$ present. $^{6}$

and baseline risk status of patients. However, rates of decline in perioperative mortality have been greatest in high HDI countries. ${ }^{9}$ Basic surgical and anaesthetic care is fundamental to providing effective primary care at the district and rural level. ${ }^{10}{ }^{11}$ Renewed emphasis on the need for essential surgical services as an integral part of achieving universal healthcare has been the focus of the WHO's Emergency and Essential Surgical Care (EESC) programme (http://www.who.int/surgery/en) and the Lancet Commission on Global Surgery. ${ }^{12}{ }^{13}$ Recently, the 68th WHA passed resolution A68/15 on 'Strengthening Emergency and Essential Surgical Care and Anaesthesia as a Component of Universal Health Coverage', which has catapulted the importance of essential surgery on the post-2015 sustainable goals development agenda. ${ }^{14}$ Yet, wide gaps in infrastructure, trained personnel and basic equipment in the LMIC setting are significant barriers to achieving this mandate.

The structure of health facilities in PNG is outlined in figure 2. In 2010, an estimated $20-30 \%$ of 4182 aid posts were no longer in operation, and concerns remain that this number has worsened due to challenges in maintaining a network of trained staff and supplies within

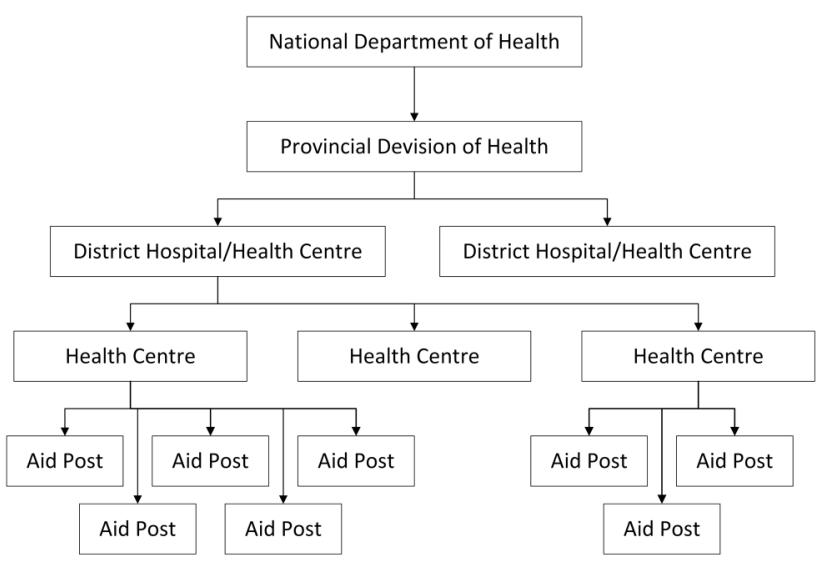

Figure 2 Structure of Papua New Guinea's healthcare system. ${ }^{4}$ remote areas with lacking roads and water transportation (table 1). ${ }^{48}$ Trauma represents $60 \%$ of surgical admissions, and accidental injury, obstetric and gynaecological, and perinatal conditions represent the third to fifth leading causes of admission to hospitals, respectively, accounting for more than 700 admissions per 100000 people in PNG. ${ }^{45}$ About 5\% of the national budget is spent on healthcare, and PNG has an estimated 0.58 health workers per 1000 population. ${ }^{416}$

Lack of access to essential surgery services contributes significantly to global morbidity and mortality. About $11-15 \%$ of the world's disability is due to surgically treatable conditions, ${ }^{11}$ yet more than two billion people lack access to even the most basic surgical availability. ${ }^{11-13} 17$ Out of the 350 million surgeries performed each year worldwide, only $3.5 \%$ are performed on the poorest $1 / 3$ of the global population. ${ }^{18}$ Injuries alone contribute to 5.7 million premature deaths annually, which outpaces the 3.8 million deaths due to malaria, AIDS and tuberculosis combined. Southeast Asia is thought to be the region of the world with the largest unmet need, accounting for 48 million disease-adjusted life-years. ${ }^{19}$

The current state of emergency and surgical capacity across PNG is inadequately understood, and precludes setting-specific benchmarks for measured action. ${ }^{415162021}$ To date, there have been no published surveys of capacity for essential emergency, surgery and anaesthesia services within PNG. The purpose of this study was to assess key indicators of capacity to provide surgical services including emergency, obstetrics and anaesthesia across PNG, in order to support future evidence-based decision-making in this area.

\section{METHODS}

In 2009, a team from PNG National Department of Health (NDOH) and the WHO EESC programme visited six health facilities in Hiri district, Rigo district, and Port Moresby General Hospital of PNG. ${ }^{15} 16 \quad 20$ These centres were chosen based on a convenience sample respresenting a number of regions, but within 
Table 1 Healthcare facilities in Papua New Guinea in $2010 .{ }^{48}$

\begin{tabular}{|c|c|c|c|c|}
\hline Category & Government & Mission or private & Total, $\mathbf{n}$ & Per cent \\
\hline National/provincial hospitals & 20 & 2 & 22 & 0.5 \\
\hline Urban clinics & 48 & 21 & 69 & 1.6 \\
\hline District/rural hospitals & 5 & 9 & 14 & 0.3 \\
\hline Health centres & 149 & 52 & 201 & 4.8 \\
\hline Health subcentres & 158 & 270 & 428 & 10.2 \\
\hline Aid posts (open) & 2672 & - & - & 63.9 \\
\hline Aid posts (closed) & 776 & - & - & 18.6 \\
\hline Total & 3828 & 354 & 4182 & - \\
\hline
\end{tabular}

limits of accessibility within a short availability of time. In addition, NDOH and WHO partnered workshops were provided at the PNG Medical Society Symposium to discuss opportunities for capacity building in essential surgical services for conditions amenable to surgical intervention, ${ }^{15}{ }^{16}{ }^{20}$ and to introduce the WHO Situational Analysis Tool (WHO SAT, available at http:// www.who.int/surgery/en).

The WHO SAT consists of a 108-item questionnaire for healthcare facilities to collect information about population served, infrastructure, human resources, interventions, equipment and supplies with a focus on emergency, trauma, obstetrics, surgery and anaesthetic services. ${ }^{22}{ }^{23}$ The WHO SAT was distributed during the workshop and during site visits. WHO SAT surveys were completed voluntarily by healthcare personnel or administrators within hospitals, health centres, or aid posts in PNG. Surveys were submitted to WHO by email, mail, or fax or through the WHO country office. Hospitals and peripheral health centres were invited to participate in a cross-sectional survey of surgery and anaesthesia capacity across PNG. The invitation was primarily disseminated during $\mathrm{NDOH}$-sponsored and WHO-sponsored workshops which included healthcare workers from many rural and urban centres. Healthcare workers and administrators who participated in the survey were also encouraged to contact other hospitals and health centres to invite them to participate.

The survey data were entered by WHO personnel into the EESC Global database.

For this study, all surveys submitted by PNG facilities up to June 2013 were collected from the WHO EESC database. Categorisation of data was planned according to the following facility types: national/provincial hospitals, district/rural hospitals, and health centres or aid posts (called 'health centres', for simplicity). The designation of health facility type was verified through direct contact or through online searches of PNG websites.

Descriptive summary statistics were planned for each of these categories of facilities, including population served by the facility, number of admissions, number of functioning operating rooms (ORs), number of surgeries performed (major and minor combined) for adults, number of surgeries performed (major and minor combined) for paediatrics (age $<15$ years), number of patients referred to other centres (due to insufficient local capacity), and distance travelled by patients to reach the facility for surgical services. In addition, details about infrastructure, equipment and supplies were summarised for each category of facility through heat map tables where green indicates uninterrupted supply, yellow indicates interrupted supply and red indicates unavailable. Human resources were characterised by facility type, with particular emphasis on availability of certified surgeons, obstetrician/gynaecologists, anaesthesiologists, general physicians or other trained nurses, health extension officers, midwives or paramedics performing surgery or anaesthesia. The proportion of facilities that indicated capacity to provide each service listed within the WHO SAT was also summarised. The tables and heat maps describe the results separately for national/provincial and district/rural hospitals (where different levels of surgery are generally expected to be provided) versus primary health centres (where surgery is not a primary aim, but rather where basic resuscitation, limited 'surgical' capabilities for first aid, burns, wounds and obstetric care would be expected).

When possible, information was validated through discussions with the submitter by email when survey data were unclear or missing. If contact was unsuccessful, other members within the facility were emailed, or members of the PNG Society for Rural and Remote Medicine were contacted for information. Through this network, most of the missing and unclear data were successfully ascertained. Any remaining data not reported is indicated by 'NR' in the summary tables.

Proportions were summarised as percentage facilities with uninterrupted supply for the respective infrastructure items. Medians and ranges were provided as a summary estimate for numeric data. Since the earlier version of SAT (before 2012) provides preset categories for numeric data (ie, 3-4, 5-10, 11-20, 21-50, etc), the median was reported in accordance with these definitions, and the range was reported as the lowest and highest possible number for the extremes of the reported ranges. Descriptive comparisons between national/provincial and district/rural centres were planned, without statistical inference. 


\section{RESULTS}

A total of 25 WHO SAT surveys were identified in the WHO database, with submission dates ranging from 2008 to 2011. After removing duplicate reports (only the most recent report from each centre was retained), there remained a total of 21 surveys including 3 national/provincial hospitals, $11 \mathrm{district} /$ rural hospitals and 7 health centres. The surveys represented a broad geographic coverage of the provinces and regions of PNG, as shown by the map in figure 3. Most hospitals in PNG are represented in this study.

Table 2 provides a description of included facilities. District/rural hospitals generally served around 100 000, 1000-2000 admissions annually, with fewer than 100 beds and 1 or 2 functioning ORs. Two national/provincial hospitals had under 10 ORs to support several thousand surgeries annually. Most hospitals provided adult and paediatric surgery. District/rural hospitals were more likely to refer a significant proportion of patients to other facilities for surgery. Some patients travel a long distance to reach referral centres $(300-700 \mathrm{~km})$. While each national/provincial hospital reported devoted emergency department (ED) and postanaesthesia care unit (PACU) areas, less than 50\% of district/rural hospitals reported having these. The number of beds and ORs per facility was not necessarily commensurate with the size of population served, number of admissions and number of surgeries performed.

\section{Infrastructure}

The heat map in table 3 reveals several gaps in basic essential elements of infrastructure (yellow and red areas), with increasing gaps in district/rural hospitals and health centres. All national/provincial hospitals had uninterrupted access to essential infrastructure, including running water, electricity, oxygen, anaesthesia machine, pulse oximetry, blood bank, laboratory work and X-ray machines. Whereas, at district/rural hospitals, most (but not all) reported uninterrupted access to running water and electricity, but fewer than $50 \%$ reported consistent access to oxygen cylinders or concentrators.

Fewer than half of district/rural hospitals had access to anaesthesia machines, and only $36 \%$ had consistent access to pulse oximetry, despite performing a large number of surgeries each year. Similarly, only $36 \%$ of centres reported consistent blood bank access. About half of district/rural hospitals had access to X-ray machines, and $72 \%$ had access to haemoglobin assay and urinalysis. Most district/rural hospitals reported multiple deficiencies in basic infrastructure, with only one district/rural hospital reporting no deficiencies. Access to oxygen cylinders or concentrators, anaesthesia machines, and blood bank were the most commonly reported infrastructure deficiencies. In general, district/ rural hospitals with the most severe infrastructure deficiencies performed relatively fewer surgeries than those reporting better infrastructure, despite the fact that they had several hundred or thousands of patient admissions annually and served a large population within PNG.

Infrastructure for facilities categorised as health centres was extremely limited, with only one reporting uninterrupted access to running water, and just over half reporting uninterrupted electricity, and less than 50\% reporting consistent access to oxygen. None had access to anaesthesia machines, pulse oximetry, blood bank or X-ray machines, despite performing up to 400 surgical procedures per year.

\section{Human resources}

National/provincial hospitals had 2-9 qualified surgeons, and were supplemented by general doctors or nurse/health extension officers who also performed surgery (table 4 ). In addition, they generally reported at least one qualified anaesthesiologist, supplemented by
Figure 3 Location of health facilities included in this study (clustering and overlaps prevent two of the pins from being visible near Port Moresby and near Kundiawa). Data for figure 3 is available under the Open Database License. The cartography is licensed as CC BY-SA. www.openstreetmap.org/ copyright.

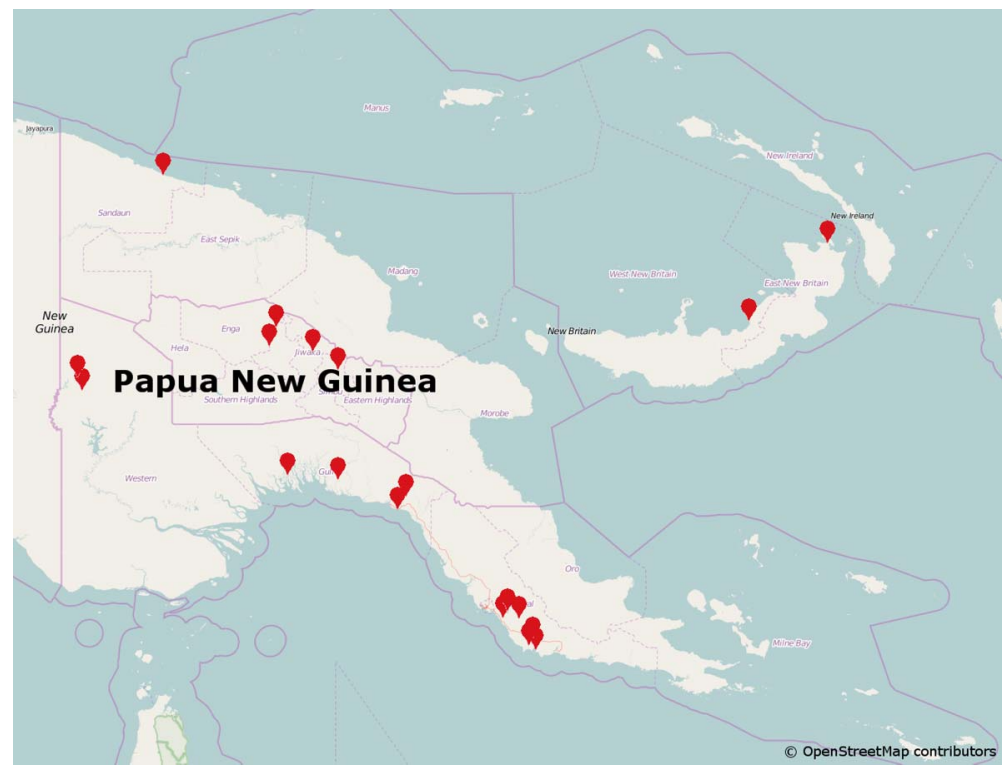


Table 2 Description of PNG healthcare facilities responding to WHO SAT Survey

\begin{tabular}{|c|c|c|c|c|c|c|c|c|c|}
\hline & $\begin{array}{l}\text { Population } \\
\text { served by this } \\
\text { facility }\end{array}$ & Beds & Admissions/year & \#ORs & $\begin{array}{l}\text { Surgeries/ } \\
\text { year }\end{array}$ & $\begin{array}{l}\text { Paediatric } \\
\text { surgeries/year }\end{array}$ & $\begin{array}{l}\text { Referred out } \\
\text { for surgery/ } \\
\text { year }\end{array}$ & $\begin{array}{l}\text { Kilometres } \\
\text { travelled to } \\
\text { this facility }\end{array}$ & $\begin{array}{l}\text { Kilometres } \\
\text { travelled if } \\
\text { referred out }\end{array}$ \\
\hline \multicolumn{10}{|l|}{ N/P $(n=3)$} \\
\hline N/P \#1 & 6000000 & $501-700$ & $>5000$ & $5-10$ & $>5000$ & $201-300$ & $5-10$ & $5-10$ & $5-10$ \\
\hline N/P \#2 & 26000 & $201-300$ & $>5000$ & $3-4$ & $1001-2000$ & $81-100$ & $5-10$ & $21-50$ & NR \\
\hline N/P \#3 & 15000 & $21-50$ & $301-400$ & 2 & $51-80$ & $5-10$ & $11-20$ & $201-300$ & $81-100$ \\
\hline Median (range) & $\begin{array}{l}26000 \\
(15000-6 \mathrm{~m})\end{array}$ & $\begin{array}{l}201-300 \\
(21-700)\end{array}$ & $\begin{array}{l}>5000 \\
(301 \text { to }>5000)\end{array}$ & $\begin{array}{l}3 \text { to } 4 \\
(2-11)\end{array}$ & $\begin{array}{l}1001-2000 \\
(51 \text { to }>5000)\end{array}$ & $81-100(5-300)$ & $5-10(5-20)$ & $21-50(5-300)$ & - \\
\hline \multicolumn{10}{|c|}{ District/rural district hospitals $(n=11)$} \\
\hline $\mathrm{D} / \mathrm{R} \# 1$ & 75000 & $101-200$ & $1001-2000$ & 2 & $101-200$ & $21-50$ & $51-80$ & $11-20$ & NR \\
\hline $\mathrm{D} / \mathrm{R} \# 2$ & 100000 & $51-80$ & NR & 1 & $101-200$ & $81-100$ & $3-4$ & $51-80$ & $501-700$ \\
\hline $\mathrm{D} / \mathrm{R} \# 3$ & 30000 & $51-80$ & $1001-2000$ & 2 & $401-500$ & $11-20$ & $11-20$ & $21-50$ & NR \\
\hline $\mathrm{D} / \mathrm{R} \# 4$ & 40000 & $21-50$ & $401-500$ & 1 & $101-200$ & $51-80$ & $5-10$ & $5-10$ & $101-200$ \\
\hline $\mathrm{D} / \mathrm{R} \# 5$ & 10000 & $11-20$ & $201-500$ & 1 & $11-20$ & $5-10$ & $21-50$ & $201-300$ & $2001-5000$ \\
\hline D/R \#6 & 30000 & $51-80$ & $701-1000$ & 1 & $201-300$ & NR & $11-20$ & NR & NR \\
\hline $\mathrm{D} / \mathrm{R} \# 7$ & 122000 & $51-80$ & $2001-5000$ & 2 & $301-400$ & $81-100$ & $81-100$ & $5-10$ & $5-10$ \\
\hline $\mathrm{D} / \mathrm{R} \# 8$ & 256000 & $101-200$ & $2001-5000$ & 2 & $2001-5000$ & $201-300$ & $21-50$ & $11-20$ & NR \\
\hline $\mathrm{D} / \mathrm{R} \# 9$ & 100000 & $201-300$ & $>5000$ & 2 & $401-500$ & $81-100$ & $81-100$ & NR & $81-100$ \\
\hline $\mathrm{D} / \mathrm{R} \# 10$ & 118000 & $101-200$ & $2001-5000$ & 2 & $401-500$ & $81-100$ & $5-10$ & $51-80$ & $101-200$ \\
\hline $\mathrm{D} / \mathrm{R} \# 11$ & 100000 & $51-80$ & $1001-2000$ & 1 & $301-400$ & $21-50$ & $11-20$ & $21-50$ & $201-300$ \\
\hline Median (range) & $\begin{array}{l}100000 \\
(10000-356000)\end{array}$ & $\begin{array}{l}51-80 \\
(11-300)\end{array}$ & $\begin{array}{l}1001-2000 \\
(201 \text { to }>5000)\end{array}$ & $2(1-2)$ & $\begin{array}{l}301-400 \\
(11-5000)\end{array}$ & $81-100(5-300)$ & $21-50(3-100)$ & $21-50(5-300)$ & $201-300(5-700)$ \\
\hline \multicolumn{10}{|l|}{$\mathrm{HC} / \mathrm{AP}(\mathrm{n}=7)$} \\
\hline HC/AP \#1 & 5000 & $21-50$ & $701-1000$ & 1 & $3-4$ & 2 & $11-20$ & 2 & $21-50$ \\
\hline HC/AP \#2 & 9000 & 0 & 0 & 1 & $11-20$ & 0 & $11-20$ & $5-10$ & NR \\
\hline $\mathrm{HC} / \mathrm{AP} \# 3$ & NR & 0 & 0 & 1 & $11-20$ & 0 & $11-20$ & $5-10$ & NR \\
\hline HC/AP \#4 & NR & 0 & $21-50$ & 1 & $51-80$ & $21-50$ & $11-20$ & $21-50$ & $81-100$ \\
\hline HC/AP \#5 & NR & $5-10$ & 0 & 1 & $201-300$ & $51-80$ & $5-10$ & $81-100$ & $81-100$ \\
\hline $\mathrm{HC} / \mathrm{AP} \# 6$ & NR & 0 & 0 & 1 & $301-400$ & $201-300$ & $51-80$ & $3-4$ & $11-20$ \\
\hline HC/AP \#7 & NR & $5-10$ & $21-50$ & 1 & $301-400$ & $201-300$ & $11-20$ & $21-50$ & $51-80$ \\
\hline Median (range) & Unknown & $0(0-50)$ & $0(0-1000)$ & 1 & $51-80(3-400)$ & $21-50(0-300)$ & $11-20(5-80)$ & $5-10(2-100)$ & $51-80(11-100)$ \\
\hline
\end{tabular}


Table 3 Infrastructure

\begin{tabular}{|c|c|c|c|c|c|c|c|c|c|c|c|c|}
\hline & $\begin{array}{l}\text { Admissions/ } \\
\mathrm{yr}\end{array}$ & $\begin{array}{l}\text { Surgeries/ } \\
\mathrm{yr}\end{array}$ & $\begin{array}{l}\text { Pediatric } \\
\text { Surgeries/ } \\
\text { yr }\end{array}$ & $\begin{array}{l}\text { Running } \\
\text { Water }\end{array}$ & Electricity & $\begin{array}{l}\text { Oxygen } \\
\text { cylinder }\end{array}$ & $\begin{array}{c}\text { Oxygen } \\
\text { concentrator }\end{array}$ & $\begin{array}{l}\text { Anaesthesia } \\
\text { Machine }\end{array}$ & $\begin{array}{c}\text { Pulse } \\
\text { Oximetry }\end{array}$ & $\begin{array}{l}\text { Blood } \\
\text { Bank }\end{array}$ & $\begin{array}{c}\text { Hgb \& } \\
\text { Urinalysis }\end{array}$ & $\begin{array}{c}x \text {-ray } \\
\text { machine }\end{array}$ \\
\hline \multicolumn{13}{|c|}{ National/Provincial Hospitals $(n=3)$} \\
\hline N/P\#1 & $>5000$ & $>5000$ & $201-300$ & & & & & & & & & \\
\hline N/P \#2 & $>5000$ & $\begin{array}{l}1001- \\
2000\end{array}$ & $81-100$ & & & & & & & & & \\
\hline $\mathrm{N} / \mathrm{P} \# 3$ & $301-400$ & $51-80$ & $5-10$ & & & & & & & & & \\
\hline \multicolumn{4}{|c|}{$\begin{array}{l}\text { National/Provincial Hospitals with } \\
\text { Uninterrupted Supply }\end{array}$} & $100 \%$ & $100 \%$ & $66 \%$ & $100 \%$ & $100 \%$ & $100 \%$ & $100 \%$ & $100 \%$ & $100 \%$ \\
\hline \multicolumn{13}{|c|}{ District/Rural Hospitals ( $\mathrm{n=11}$ ) } \\
\hline $\mathrm{D} / \mathrm{R} \# 1$ & $1001-2000$ & $101-200$ & $21-50$ & & & & & & & & & \\
\hline $\mathrm{D} / \mathrm{R} \# 2$ & NR & $101-200$ & $81-100$ & & & & & & & & & \\
\hline $\mathrm{D} / \mathrm{R} \# 3$ & $1001-2000$ & $401-500$ & $11-20$ & & & & & & & & & \\
\hline $\mathrm{D} / \mathrm{R} \# 4$ & $401-500$ & $101-200$ & $51-80$ & & & & & & & & & \\
\hline $\mathrm{D} / \mathrm{R} \# 5$ & $201-500$ & $11-20$ & $5-10$ & & & & & & & & & \\
\hline $\mathrm{D} / \mathrm{R} \# 6$ & $701-1000$ & $201-300$ & NR & & & & & & & & & \\
\hline $\mathrm{D} / \mathrm{R} \# 7$ & $\begin{array}{l}2001- \\
5000\end{array}$ & $301-400$ & $81-100$ & & & & & & & & & \\
\hline $\mathrm{D} / \mathrm{R} \# 8$ & $\begin{array}{l}2001- \\
5000\end{array}$ & $\begin{array}{l}2001- \\
5000\end{array}$ & 201-300 & & & & & & & & & \\
\hline $\mathrm{D} / \mathrm{R} \# 9$ & $>5000$ & $401-500$ & $81-100$ & & & & & & & & & \\
\hline $\begin{array}{l}\mathrm{D} / \mathrm{R} \\
\# 10\end{array}$ & $\begin{array}{l}2001- \\
5000\end{array}$ & $401-500$ & $81-100$ & & & & & & & & & \\
\hline $\begin{array}{l}\mathrm{D} / \mathrm{R} \\
\# 11\end{array}$ & $1001-2000$ & $301-400$ & $21-50$ & & & & & & & & & \\
\hline \multicolumn{4}{|c|}{$\begin{array}{l}\text { District/Rural Hospitals with } \\
\text { Uninterrupted Supply }\end{array}$} & $91 \%$ & $91 \%$ & $45 \%$ & $36 \%$ & $45 \%$ & $36 \%$ & $36 \%$ & $72 \%$ & $55 \%$ \\
\hline \multicolumn{13}{|c|}{ Health Centres/Aid Posts ( $n=7$ ) } \\
\hline $\begin{array}{l}\mathrm{HC} / \mathrm{AP} \\
\# 1\end{array}$ & $701-1000$ & $3-4$ & 2 & & & & & & & & & \\
\hline $\begin{array}{l}\mathrm{HC} / \mathrm{AP} \\
\# 2\end{array}$ & 0 & $11-20$ & 0 & & & & & & & & & \\
\hline $\begin{array}{l}\mathrm{HC} / \mathrm{AP} \\
\# 3\end{array}$ & 0 & $11-20$ & 0 & & & & & & & & & \\
\hline $\begin{array}{l}\mathrm{HC} / \mathrm{AP} \\
\# 4\end{array}$ & $21-50$ & $51-80$ & $21-50$ & & & & & & & & & \\
\hline $\begin{array}{l}\mathrm{HC} / \mathrm{AP} \\
\# 5\end{array}$ & 0 & $201-300$ & $51-80$ & & & & & & & & & \\
\hline $\begin{array}{l}\mathrm{HC} / \mathrm{AP} \\
\# 6\end{array}$ & 0 & $301-400$ & $201-300$ & & & & & & & & & \\
\hline $\begin{array}{l}\mathrm{HC} / \mathrm{AP} \\
\# 7\end{array}$ & $21-50$ & $301-400$ & $201-300$ & & & & & & & & & \\
\hline \multicolumn{4}{|c|}{ Health Centres with Uninterrupted supply } & $14 \%$ & $57 \%$ & $43 \%$ & $0 \%$ & $0 \%$ & $0 \%$ & $0 \%$ & $43 \%$ & $0 \%$ \\
\hline \multicolumn{13}{|c|}{$\begin{array}{l}\text { Green = uninterrupted supply; Yellow = interrupted supply; Red = unavailable; N/P = National/Provincial } \\
\text { hospitals; D/R = District/Rural Hospital; HC/AP = Health Centre/Aid-Posts }\end{array}$} \\
\hline
\end{tabular}

general doctors or nurse/health extension officers performing anaesthesia, and at least one obstetrician/gynecologist. In contrast, most district/rural hospitals did not have qualified surgeons, obstetrician/gynaecologists or anaesthesiologists. In general, district/rural hospitals were more likely to rely on general doctors or nurse/ health extension officers to perform surgery and anaesthesia, some of whom were not certified. Most district/ rural hospitals had at least one paramedic/midwife, with one hospital reporting up to eight paramedic/midwives. The number of healthcare workers was low relative to patient admissions and surgeries performed. At the health centres, there were few qualified healthcare personnel. The health centres were more likely to rely on health extension officers and/or paramedics/midwives (ie, community health workers).

\section{Surgical and anaesthetic interventions}

National/provincial hospitals indicated uniform capacity to provide general, regional or spinal anaesthesia, while district/rural hospitals indicated less capacity to provide general anaesthesia. Health centres generally did not report capacity to provide anaesthesia, with the exception of one centre which provides ketamine sedation (table 5). Routine use of guidelines for surgery, anaesthesia and pain were reported in $66 \%$ of national/provincial hospitals, and only $20 \%$ of district/rural hospitals, and none of the health centres.

National/provincial hospitals reported capacity to provide most procedures listed in the WHO SAT (table 5), although one of three national/provincial hospitals reported lack of capacity for cricothyroidotomy/tracheostomy, clubfoot, cleft lip and neonatal surgery. In general, 


\begin{tabular}{|c|c|c|c|c|c|c|c|c|c|c|c|}
\hline & $\begin{array}{l}\text { Admissions/ } \\
\text { year }\end{array}$ & $\begin{array}{l}\text { Surgeries/ } \\
\text { year }\end{array}$ & $\begin{array}{l}\text { Paediatric } \\
\text { surgeries/ } \\
\text { year }\end{array}$ & $\begin{array}{l}\text { Surgeons } \\
\text { (FT or PT) }\end{array}$ & $\begin{array}{l}\text { Obstetrician/ } \\
\text { gynaecologists } \\
\text { (FT or PT) }\end{array}$ & $\begin{array}{l}\text { General } \\
\text { MDs } \\
\text { performing } \\
\text { surgery } \\
\text { (FT or PT) } \\
\end{array}$ & $\begin{array}{l}\text { Nurse or } \\
\text { medical } \\
\text { officer } \\
\text { performing } \\
\text { surgery }\end{array}$ & $\begin{array}{l}\text { Anaesthesio- } \\
\text { logists } \\
\text { (FT or PT) }\end{array}$ & $\begin{array}{l}\text { General } \\
\text { MDs } \\
\text { performing } \\
\text { anaesthesia } \\
\text { (FT or PT) }\end{array}$ & $\begin{array}{l}\text { Nurse or } \\
\text { medical } \\
\text { officer } \\
\text { performing } \\
\text { anaesthesia }\end{array}$ & $\begin{array}{l}\text { Paramedics/ } \\
\text { midwives }\end{array}$ \\
\hline \multicolumn{12}{|l|}{$N / P(n=3)$} \\
\hline N/P \#1 & $>5000$ & $>5000$ & $201-300$ & 9 & 5 & 10 & 0 & 14 & 4 & 4 & 0 \\
\hline N/P \#2 & $>5000$ & $1001-2000$ & $81-100$ & 3 & 1 & 1 & 1 & 2 & 0 & 0 & 0 \\
\hline N/P \#3 & $301-400$ & $51-80$ & $5-10$ & 2 & 0 & 4 & 4 & 1 & 1 & 1 & 2 \\
\hline \multicolumn{4}{|c|}{ Median (range) number in N/P } & $3(2-9)$ & $1(0-5)$ & $4(1-10)$ & $1(0-5)$ & $2(1-14)$ & $1(0-4)$ & $1(0-4)$ & $0(0-2)$ \\
\hline \multicolumn{12}{|c|}{$D / R(n=11)$} \\
\hline $\mathrm{D} / \mathrm{R} \# 1$ & $1001-2000$ & $101-200$ & $21-50$ & 1 & 0 & 2 & 6 & 0 & 0 & 2 & 1 \\
\hline $\mathrm{D} / \mathrm{R} \# 2$ & NR & $101-200$ & $81-100$ & 0 & 0 & 1 & 1 & 0 & 0 & 1 & 0 \\
\hline D/R \#3 & $1001-2000$ & $401-500$ & $11-20$ & 2 & 0 & 1 & 0 & 0 & 0 & 1 & 8 \\
\hline $\mathrm{D} / \mathrm{R} \# 4$ & $401-500$ & $101-200$ & $51-80$ & 0 & 0 & 2 & 0 & 0 & 2 & 1 & 0 \\
\hline $\mathrm{D} / \mathrm{R}$ \#5 & $201-500$ & $11-20$ & $5-10$ & 0 & 0 & 2 & 1 & 0 & 0 & 1 & 1 \\
\hline $\mathrm{D} / \mathrm{R} \# 6$ & $701-1000$ & $201-300$ & NR & 0 & 0 & 2 & 0 & 0 & 2 & 0 & 0 \\
\hline $\mathrm{D} / \mathrm{R} \# 7$ & $2001-5000$ & $301-400$ & $81-100$ & 1 & 1 & 1 & 1 & 1 & 1 & 2 & 3 \\
\hline $\mathrm{D} / \mathrm{R} \# 8$ & $2001-5000$ & $2001-5000$ & $201-300$ & 1 & 0 & 6 & 1 & 0 & 0 & 2 & 2 \\
\hline $\mathrm{D} / \mathrm{R} \# 9$ & $>5000$ & $401-500$ & $81-100$ & 0 & 1 & 1 & 1 & 0 & 0 & 2 & 4 \\
\hline D/R \#10 & $2001-5000$ & $401-500$ & $81-100$ & 0 & 0 & 2 & 2 & 0 & 0 & 1 & 1 \\
\hline D/R \#11 & 1001-2000 & $301-400$ & $21-50$ & 0 & 0 & 1 & 0 & 0 & 0 & 0 & 5 \\
\hline \multicolumn{4}{|c|}{$\begin{array}{l}\text { Median (range) number in rural/district hospitals } \\
\operatorname{HC} / A P(n=7)\end{array}$} & $0(0-2)$ & $0(0-1)$ & $1(1-6)$ & $1(0-6)$ & $0(0-1)$ & $0(0-2)$ & $1(0-2)$ & $1(0-5)$ \\
\hline HC/AP \#1 & $701-1000$ & $3-4$ & 2 & 0 & 0 & 0 & 0 & 0 & 0 & 0 & 1 \\
\hline HC/AP \#2 & 0 & $11-20$ & 0 & 0 & 0 & 0 & 0 & 0 & 0 & 1 & 2 \\
\hline HC/AP \#3 & 0 & $11-20$ & 0 & 0 & 0 & 0 & 1 & 0 & 0 & 0 & 0 \\
\hline HC/AP \#4 & $21-50$ & $51-80$ & $21-50$ & 0 & 0 & 0 & 1 & 0 & 0 & 0 & 0 \\
\hline HC/AP \#5 & 0 & 201-300 & $51-80$ & 0 & 0 & 0 & 1 & 0 & 0 & 1 & 2 \\
\hline HC/AP \#6 & 0 & $301-400$ & 201-300 & 0 & 0 & 0 & 0 & 0 & 0 & 0 & 3 \\
\hline HC/AP \#7 & $21-50$ & $301-400$ & $201-300$ & 0 & 0 & 2 & 1 & 0 & 0 & 1 & 1 \\
\hline \multicolumn{4}{|c|}{ Median (range) number in HC/AP } & 0 & 0 & $0(0-2)$ & $1(0-1)$ & 0 & 0 & $0(0-1)$ & $1(3-3)$ \\
\hline
\end{tabular}

$\mathrm{D} / \mathrm{R}$, district/rural hospitals; FT, full-time; HC/AP, health centres/aid posts; N/P, national/provincial hospitals; NR, not reported; PT, part-time. 
Table 5 Interventions (\% of facilities reporting capacity to provide selected interventions)

\begin{tabular}{|c|c|c|c|}
\hline & $\begin{array}{l}\text { National/provincial } \\
\text { hospital, } n=3(\%)\end{array}$ & $\begin{array}{l}\text { District/rural hospital, } \\
\mathrm{n}=11(\%)\end{array}$ & $\begin{array}{l}\text { Health centre/aid } \\
\text { posts, } n=7(\%)\end{array}$ \\
\hline \multicolumn{4}{|l|}{ Anaesthesia } \\
\hline General anaesthesia & 100 & 64 & 0 \\
\hline Spinal anaesthesia & 100 & 91 & 0 \\
\hline Regional anaesthesia & 100 & 100 & 0 \\
\hline Ketamine anaesthesia & 66 & 100 & 14 \\
\hline \multicolumn{4}{|l|}{ Surgical procedures } \\
\hline Resuscitation* & 100 & 100 & 14 \\
\hline Cricothyroidotomy/tracheostomy & 66 & 64 & 0 \\
\hline Chest tube & 100 & 100 & 0 \\
\hline Caesarean section & 100 & 82 & 0 \\
\hline Dilation and curettage & 100 & 100 & 0 \\
\hline Obstetric fistula repair & 66 & 36 & 0 \\
\hline Hydrocele & 100 & 82 & 0 \\
\hline Cystostomy & 100 & 55 & 0 \\
\hline Laparotomy & 100 & 64 & 0 \\
\hline Tubal ligation & 100 & 91 & 0 \\
\hline Hernia repair, strangulated & 100 & 72 & 0 \\
\hline Hernia repair, congenital & 100 & 64 & 0 \\
\hline Cleft lip and palate repair & 66 & 18 & 0 \\
\hline Neonatal surgery $\dagger$ & 66 & 27 & 0 \\
\hline Club foot repair & 66 & 55 & 0 \\
\hline Fracture reduction, closed & 100 & 100 & 57 \\
\hline Fracture reduction, open & 100 & 64 & 14 \\
\hline Amputation & 100 & 72 & 14 \\
\hline Joint dislocation & 100 & 100 & 71 \\
\hline Male circumcision & 100 & 100 & 0 \\
\hline Urethral stricture dilator & 66 & 55 & 0 \\
\hline Appendectomy & 100 & 64 & 0 \\
\hline Acute burns & 100 & 100 & 86 \\
\hline Contracture release, skin grafting & 100 & 91 & 0 \\
\hline Drainage of osteomyelitis or septic arthritis & 100 & 91 & 0 \\
\hline Abscess incision and drainage & 100 & 100 & 100 \\
\hline Wound debridement & 100 & 100 & 71 \\
\hline Suturing & 100 & 100 & 100 \\
\hline Foreign body removal & 100 & 100 & 29 \\
\hline Biopsy & 100 & 82 & 14 \\
\hline Cataract surgery & 66 & 18 & 0 \\
\hline
\end{tabular}

${ }^{*}$ Resuscitation-airway, haemorrhage, peripheral intravenous access, peripheral venous cut down.

†Neonatal surgery-abdominal wall defect, colostomy, imperforate anus, intussusception.

district/rural hospitals reported capacity to provide resuscitation, chest tube insertion, dilation and curettage, joint dislocation, male circumcision, acute burn management, skin grafting and contractures treatment, abscess incision and drainage, wound debridement, suturing, foreign body removal, drainage of osteomyelitis or septic arthritis, and closed fracture reduction. Capacity to provide caesarean section was indicated by $82 \%$ of national/provincial hospitals, and tubal ligation by $91 \%$, but only $36 \%$ had capacity to provide obstetrical fistula repairs. Few district/rural hospitals provided neonatal surgery, cleft lip repair or clubfoot repair. Health centres indicated limited capacity for most types of procedures. Of particular concern is the lack of reported capacity for basic resuscitation at the health centres.

\section{Equipment and supplies}

The heat map in table 6 reveals frequent deficiencies in essential equipment and supplies, which progressively increases from national/provincial hospitals, to district/ rural hospitals, and health centres. None of the facility types reported consistent access to all of the listed equipment. Even at the national/provincial hospitals, frequent shortages in essential equipment were reported. At district/rural hospitals, frequent deficiencies were reported for oxygen delivery, cricothyroidotomy supplies, intravenous fluid delivery, sterilisation and personal protective equipment including gloves. At the health centres, almost all categories of equipment and supplies were frequently or completely unavailable, including all basic resuscitation equipment. 
Table 6 Equipment and supplies

\begin{tabular}{|c|c|c|c|c|c|c|c|c|c|c|c|c|c|c|c|}
\hline & $\begin{array}{c}\text { Admissions } \\
\text { per yr }\end{array}$ & 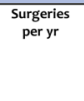 & $\begin{array}{c}\text { Pediatric } \\
\text { Surgereser } \\
\text { yr }\end{array}$ & $\begin{array}{l}\text { Resuscitator } \\
\text { bag \& mask } \\
\text { (adult) }\end{array}$ & 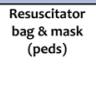 & $\begin{array}{l}\text { Oxygen } \\
\text { and Mask } \\
\text { Tulibs to } \\
\text { connect } \\
\text { oxygen }\end{array}$ & $\begin{array}{c}\text { Sterile } \\
\text { Disposabble } \\
\text { Nededles }\end{array}$ & $\begin{array}{l}\text { Stetho- } \\
\text { scope }\end{array}$ & $\begin{array}{l}\text { Crycothyroidotomy } \\
\text { set }\end{array}$ & $\begin{array}{l}\text { Oropharyngeal } \\
\text { airway (adult) }\end{array}$ & $\begin{array}{l}\text { Oropharyngeal } \\
\text { airway (peds) }\end{array}$ & Sterilizer & $\begin{array}{l}\text { Sterile } \\
\text { Gloves }\end{array}$ & $\begin{array}{l}\text { Protective } \\
\text { face mask }\end{array}$ & $\begin{array}{l}\text { IV fluid set and } \\
\text { bags }\end{array}$ \\
\hline \multicolumn{16}{|c|}{ National/Provincial Hospitals $(\mathrm{n}=3$ ) } \\
\hline $\mathrm{N} / \mathrm{P} \# 1$ & $>5000$ & $>5000$ & $201-300$ & & & & & & & & & & & & \\
\hline N/P\#2 & $>5000$ & $\begin{array}{l}1001- \\
2000\end{array}$ & 81-100 & & & & & & & & & & & & \\
\hline $\mathrm{N} / \mathrm{P} \mathrm{H}_{3}$ & $301-400$ & $51-80$ & $5-10$ & & & & & & & & & & & & \\
\hline \multicolumn{4}{|c|}{$\begin{array}{r}\text { National/Provincial Hosp with } \\
\text { Uninterrupted Supply }\end{array}$} & $33 \%$ & $33 \%$ & $33 \%$ & $66 \%$ & $33 \%$ & $33 \%$ & $33 \%$ & $33 \%$ & $33 \%$ & $33 \%$ & $66 \%$ & $66 \%$ \\
\hline \multicolumn{16}{|c|}{ District/Rural Hospitals $(n=11)$} \\
\hline $\mathrm{D} / \mathrm{R} \# 1$ & $\begin{array}{l}1001- \\
2000 \\
\end{array}$ & $101-200$ & $21-50$ & & & & & & & & & & & & \\
\hline $\mathrm{D} / \mathrm{R} \# 2$ & - & $101-200$ & $81-100$ & & & & & & & & & & & & \\
\hline$D / R \# 3$ & $\begin{array}{l}1001- \\
2000 \\
\end{array}$ & $401-500$ & $11-20$ & & & & & & & & & & & & \\
\hline $\mathrm{D} / \mathrm{R} \# 4$ & $401-500$ & $101-200$ & $51-80$ & & & & & & & & & & & & \\
\hline $\mathrm{D} / \mathrm{R} \# \mathbf{5}$ & $201-500$ & $11-20$ & $5-10$ & & & & & & & & & & & & \\
\hline $\mathrm{D} / \mathrm{R} \# 6$ & $\begin{array}{l}701- \\
1000 \\
\end{array}$ & $201-300$ & 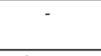 & & & & & & & & & & & & \\
\hline $\mathrm{D} / \mathrm{R} \# 7$ & $\begin{array}{ll}2001- \\
5000\end{array}$ & $301-400$ & 81-100 & & & & & & & & & & & & \\
\hline $\mathrm{D} / \mathrm{R} \# 8$ & $\begin{array}{l}2001- \\
5000\end{array}$ & $\begin{array}{l}2001- \\
5000 \\
\end{array}$ & $201-300$ & & & & & & & & & & & & \\
\hline $\mathrm{D} / \mathrm{R} \# 9$ & $>5000$ & $401-500$ & 81-100 & & & & & & & & & & & & \\
\hline $\begin{array}{l}\mathrm{D} / \mathrm{R} \\
\# 10\end{array}$ & $\begin{array}{l}2001- \\
5000\end{array}$ & $401-500$ & 81-100 & & & & & & & & & & & & \\
\hline $\mathrm{D} / \mathrm{R} \# 11$ & $\begin{array}{l}1001- \\
2000 \\
\end{array}$ & $301-400$ & $21-50$ & & & & & & & & & & & & \\
\hline \multicolumn{4}{|c|}{$\begin{array}{r}\text { District/Rural Hospitals with Uninterrupted } \\
\text { Supply }\end{array}$} & $64 \%$ & $72 \%$ & $27 \%$ & $72 \%$ & $64 \%$ & $27 \%$ & $82 \%$ & $82 \%$ & $55 \%$ & $55 \%$ & $72 \%$ & $45 \%$ \\
\hline \multicolumn{16}{|c|}{ Health Centres/Aid Posts $(\mathrm{n}=6)$} \\
\hline $\begin{array}{l}\mathrm{HC} / \mathrm{AP} \\
\# 1\end{array}$ & $\begin{array}{r}701- \\
1000 \\
\end{array}$ & 3-4 & 2 & & & & & & & & & & & & \\
\hline$\#$ HC/AP & 0 & $11-20$ & o & & & & & & & & & & & & \\
\hline HC/AP & 0 & 11-20 & 0 & & & & & & & & & & & & \\
\hline $\begin{array}{ll}\mathrm{HC} / \mathrm{AP} \\
\# 4\end{array}$ & $21-50$ & $51-80$ & $21-50$ & & & & & & & & & & & & \\
\hline $\begin{array}{ll}\mathrm{HC} / \mathrm{AP} \\
\# 5\end{array}$ & 0 & $201-300$ & $51-80$ & & & & & & & & & & & & \\
\hline HC/AP & 0 & $301-400$ & $201-300$ & & & & & & & & & & & & \\
\hline$\# \mathrm{HC} / \mathrm{AP}$ & $21-50$ & $301-400$ & $201-300$ & & & & & & & & & & & & \\
\hline \multicolumn{4}{|c|}{ HC/AP with Uninterrupted supply } & o\% & $0 \%$ & $0 \%$ & $71 \%$ & $43 \%$ & $0 \%$ & $0 \%$ & $0 \%$ & $0 \%$ & $14 \%$ & $14 \%$ & $14 \%$ \\
\hline & & ospitals; & $R=$ Distric & $\begin{array}{l}\text { oply; Ye } \\
\text { ural Ho }\end{array}$ & $\begin{array}{l}=\text { inter } \\
; \mathrm{HC} / \mathrm{A}\end{array}$ & ealth & $\begin{array}{l}\text {; Red }= \\
\text { tre/Aid }\end{array}$ & osts & & & & & & & \\
\hline
\end{tabular}

\section{DISCUSSION}

While much progress has been made in healthcare provision in recent years in PNG, important deficiencies persist which severely restrict access to essential surgery and anaesthesia services across the country. Rural facilities, including district/rural hospitals and primary health centres/aid posts, that are physically closest to where $80 \%$ of the population resides deal with the most severe restrictions in infrastructure, supply and human resources. One of the most striking findings is the lack (14\%) of even basic resuscitative and primary trauma capacity in peripheral primary care centres, including basic airway and first aid equipment, which could potentially save more lives than more advanced surgical services per se. Another striking finding was that, even when all types of facilities were considered in aggregate, no health facility reported having all infrastructure required to provide essential surgical services, despite providing thousands of surgeries annually. Many facilities manage to provide emergency and surgical services without a consistent supply of electricity, running water, oxygen, anaesthesia machines, pulse oximeters, blood bank access, intravenous fluid resuscitation and personal protective equipment. District/rural hospitals reported provision of most types of anaesthesia $(100 \%$ ketamine, $100 \%$ regional, $91 \%$ spinal, $64 \%$ general), while most primary health centres did not report anaesthetic capacity (with the exception of $14 \%$ providing ketamine), which raises important concerns about the lack of available anaesthesia for initial management of trauma and orthopaedic emergencies in the peripheral regions. While most facilities reported capability for primary obstetric care, including most peripheral centres, the near absence of basic airway equipment and intravenous resuscitative supplies in the peripheral primary care health centres remains of concern for the provision of safe obstetric care.

This survey provides a clear picture of the state of deficiencies across healthcare facilities, which has likely contributed to the persistently low performance on key Millennium Development Goals (MDGs; high maternal and child mortality, especially in the rural areas, where rates are known to be twofold higher than in urban areas of PNG), ${ }^{4}$ and will preclude achievement of equitable and efficient universal healthcare in PNG in the post-2015 development agenda. ${ }^{14}{ }^{24}$ Deficiencies in 
capacity and access result from a combination of barriers including geospatial (PNG is comprised of primarily rural populations spread over 300 islands), financial (public financing of healthcare in PNG is still in development), infrastructural (lacking roads and waterway services to connect the rural and urban areas), together with other factors that have not been well characterised (800 distinct cultures and languages exist within PNG, and a significant proportion of peripheral aid posts remain abandoned). ${ }^{2-5}$

While this study provides only a partial snap shot of the system, it provides a level of detail that has never before been published for PNG. ${ }^{25}$ Nevertheless, the results should be interpreted in light of the typical limitations associated with survey data. Given the formidable challenges including geographical and expense of achieving a complete and formalised representation of all facilities country wide, this study relied on a nonrandom convenience sample from volunteer submission of the WHO SAT from the health facilities invited to participate. Accordingly, there will be deficiencies in representativeness. Another severe limitation of the survey is that it is derived from reports and estimates provided by the respondents, rather than by direct inspection of the facilities for infrastruction, human resources and supplies. Perhaps the most important limitation of this survey is the fact that only infrastructure and activity is measured. Since clinical outcomes are not measured, there is no opportunity to correlate the deficiencies in infrastructure and human resources with clinical or population-based outcomes. However, we know from previous studies within $\mathrm{PNG}^{26}$ and other LMICs $^{9}{ }^{27-30}$ that deficits in health infrastructure, medical technology and integration of resources to provide surgery have been consistently correlated with mortality, and that intervention to alleviate these deficiencies has a tangible impact on mortality. ${ }^{26}$

While the magnitude of restrictions in surgical and anaesthesia capacity experienced by PNG is only indirectly informed by this study, the emerging picture of greatest restrictions to those who need it most represents a specific call to action. Reducing maternal and child mortality will require essential surgery to be bolstered through sequenced steps to address the facility infrastructure and trained human resources to address common debilitating conditions in PNG. ${ }^{31}{ }^{32}$ In PNG, deaths due to trauma, accidents, obstetric and neonatal conditions considered together represent a high proportion of life lost which rivals that of deaths due to HIV/ AIDS, malaria and pneumonia. Many of these deaths could be prevented through essential surgery, if it was available. Beyond premature deaths, there exists a myriad of common conditions for which surgical services would reduce morbidity, including obstetric fistulas, maternal haemorrhage, clubfoot, cleft lip and palate, acute abdominal conditions, hernias, cataracts, malignant and benign tumours, ruptured spleen (due to malaria), burns, contractures, fractures, and trauma complications, male circumcision (HIV transmission reduction), among others. ${ }^{16} 33$

The WHO SAT survey tool, as well as other surveys developed for similar purposes including the Surgeons Overseas Personnel, Infrastructure, Procedures, Equipment and Supplies (PIPES) survey, and the Harvard Humanitarian Initiative (HHI) survey tool have been applied in a number of developing countries, with similar conclusions regarding the severe gaps in infrastructure, human resources and supplies in various regions of the world. ${ }^{34-40}$ Although surgery is one of the most cost-effective interventions in LMICs, ${ }^{11}{ }^{41}$ perceptions remain that it is too expensive and complex to provide in austere settings. ${ }^{16}$ Greater efforts should be devoted to prioritising essential on the political agenda and for aid providers, ${ }^{9}{ }^{42}$ since simple life-altering procedures can bring greater value for money than other commonly supported public health interventions in LMICs. ${ }^{41}$

The government of PNG has taken important steps to place renewed focus on strengthening the healthcare system, after initial gains in the 1990s were partly defeated by setbacks in progress, revealed by 'brain drain' of qualified healthcare workers from remote areas to urban areas or out-of-country, and the closing of several aid posts. ${ }^{4} 43$ PNG has an estimated 0.58 healthcare workers per 1000 people, and fewer than 1 qualified anaesthetic provider per 100000 , which is at the lowest end of recent global surveys. ${ }^{41730343544}$ To put this into perspective, in the USA and the UK there is an estimated ratio of $1: 4000$ or 1:5000 of anaesthetic providers per capita. ${ }^{45}$

Despite much progress and many successes to be celebrated in PNG (ie, increased oxygen supply, medical records and clinical guidelines use, health extension officers and community health workers, medical residency and surgical training networks), ${ }^{432} 43$ in order to achieve post-2015 development targets, increasing essential surgical services will need to become a high priority to operationalise the National Health Plan. While the National Health Plan does not address surgery and anaesthesia services specifically, this should be encouraged. Without rehabilitation of current facilities to be outfitted with basic infrastructure including electricity, water and essential supplies, along with functional ORs, EDs, PACUs, adequately resourced with essential equipment and with trained healthcare workers at the rural level, the Vision 2050 and the post-2015 development agenda will remain theory rather than a reality. ${ }^{44} 45$

\section{CONCLUSIONS}

Despite important progress in healthcare provision in PNG, capacity for essential surgery is severely limited and unevenly provided due to limitations in physical infrastructure, human resources, and basic equipment and supplies. Appropriate solutions to these gaps should be sought through collaborative and context-sensitive approaches, and with a degree of urgency that is 
commensurate with the severity of lives lost and disability disproportionately affecting the livelihoods of the most disadvantaged.

\section{Author affiliations}

${ }^{1}$ Department of Anesthesia \& Perioperative Medicine, Medical Evidence, Decision Integrity \& Clinical Impact (MEDICI) Centre, Schulich School of Medicine \& Dentistry, Western University, London, Ontario, Canada ${ }^{2}$ Department of Epidemiology \& Biostatistics, Schulich School of Medicine \& Dentistry, Western University, London, Ontario, Canada

${ }^{3}$ National Department of Health (NDoH), National Health Service Standards, Waigani, Papua New Guinea

${ }^{4}$ Emergency \& Essential Surgical Care (EESC) Program, Service Delivery and Safety Department (SDS), Health Systems \& Innovation, World Health Organisation-HQ, Geneva, Switzerland

${ }^{5}$ Kompiam District Hospital, Mount Hagen, Papua New Guinea

${ }^{6}$ University of the West of England, Bristol, UK

${ }^{7}$ WHO Representation PNG, AOPI Center, Boroko, Papua New Guinea

Twitter Follow Jennifer Vergel de Dios at @yennyoliver

Acknowledgements The authors would like to acknowledge the healthcare workers and administrators who provided the data for this study. The authors would also like to acknowledge the support of the Papua New Guinea National Department of Health and the PNG Society for Rural and Remote Medicine in providing opportunity for site visits, and hosting the GIEESC workshop. They also would like to acknowledge Jessica Moodie (MEDICI Librarian) for her support in this research.

Contributors DM, MNC, GT and WA-K designed the study concept. DM, GT, JF and WA-K contributed to data collection and oversight. JM developed the statistical methods and approach to data presentation with input from JVdD, MNC and DC. JM analysed the data and prepared the results, which was verified by all co-authors. All authors contributed to the interpretation of the results. JM and JVdD prepared the first draft of the report, and all other authors contributed to the subsequent and final drafts.

Funding This research received no specific grant from any funding agency in the public, commercial or not-for-profit sectors.

Competing interests None declared.

Provenance and peer review Not commissioned; externally peer reviewed.

Data sharing statement Data for this study are available from the authors on request.

Open Access This is an Open Access article distributed in accordance with the Creative Commons Attribution Non Commercial (CC BY-NC 4.0) license, which permits others to distribute, remix, adapt, build upon this work noncommercially, and license their derivative works on different terms, provided the original work is properly cited and the use is non-commercial. See: http:// creativecommons.org/licenses/by-nc/4.0/

\section{REFERENCES}

1. United Nations Statistics Division: Papua New Guinea. [Internet]. 2014 (6 June 2014). http://data.un.org/CountryProfile.aspx? crName=Papua\%20New\%20Guinea

2. WHO Western Pacific Region: Papua New Guinea-statistics summary (2002 - present). http://apps.who.int/gho/data/node. country.country-PNG (accessed 6 Jun 2014).

3. The World Bank: Papua New Guinea Data. http://data.worldbank. org/country/papua-new-guinea (accessed 6 Jun 2014).

4. Government of Papua New Guinea. National Health Plan 20112010, Volume 1 Policies and Strategies. 2010 (11 April 2013). http:// www.wpro.who.int/countries/png/PNGNHP_Part1.pdf

5. World Bank. "World Bank data on urbanisation." World Development Indicators. World Bank. 2005. Archived from the original on 2009-02-03 (accessed 6 Jun 2014).

6. United Nations Development Programme. Human development reports. http://www.hdr.undp.org/en/data (accessed 6 Jun 2014).
7. Australian Department of Foreign Affairs and Trade. 9 March 2012. Raising the profile of PNG in Australia" (accessed 29 May 2014).

8. World Health Organization (WHO) and National Department of Health (NDoH) of PNG. Papua New Guinea Health Service Delivery Profile. 2012. http://www.wpro.who.int/health_services/service delivery_profile_papua_new_guinea.pdf (accessed 6 Jun 2014).

9. Bainbridge D, Martin J, Arango M, et al., Evidence-based Peri-operative Clinical Outcomes Research Group. Perioperative and anaesthetic-related mortality in developed and developing countries: a systematic review and meta-analysis. Lancet 2012;380: 1075-81.

10. Farmer P, Kim J. Surgery and global health: a view from beyond the OR. World J Surg 2008;32:533-6.

11. Debas HT, Gosselin R, McCord C, et al. Surgery. In: Debas HT, Gosselin R, McCord C, et al. eds. Disease control priorities in developing countries. 2nd edn. New York, NY: Oxford University Press, 2006:1245-60.

12. Bickler SW, Spiegel D. Improving surgical care in low- and middle-income countries: a pivotal role for the World Health Organization. World J Surg 2010;34:386-90.

13. Meara JG, Hagander L, Leather AJ. Surgery and global health: a Lancet Commission. Lancet 2014;383:12-13.

14. World Health Organization. Strengthening emergency and essential surgical care and anaesthesia as a component of universal health coverage. http://apps.who.int/gb/ebwha/pdf_files/WHA68/A68_ R15-en.pdf?ua=1 (accessed 30 May 2015).

15. World Health Organization. Fourth Meeting of the WHO Global Initiative for Emergency and Essential Surgical Care (GIEESC) 2011. http://www.who.int/surgery/globalinitiative/WHO_GIEESC_ MeetingReportNov2011.pdf (accessed 11 Apr 2013).

16. Adu-Krow W. Presentation at the session VI. Plenary session: generating political priority for EESC. World Health Organization (WHO), Emergency and Essential Surgical Care (EESC) Programme. Fifth Meeting of the World Health Organization Global Initiative for Emergency and Essential Surgical Care (GIEESC). Hosted by the Ministry of Health of the Republic of Trinidad and Tobago; Port of Spain, 14-15 October 2013. http://www.who.int/ surgery/globalinitiative/gieesc_biennialmeet/en/

17. Funk LM, Weiser TG, Berry WR, et al. Global operating theatre distribution and pulse oximetry supply: an estimation from reported data. Lancet 2010;376:1055-61.

18. Weiser TG, Regenbogen SE, Thompson KD, et al. An estimation of the global volume of surgery: a modeling strategy based on available data. Lancet 2008;372:139-44.

19. Lopez AD, Mathers CD, Ezzati M, et al. Global and regional burden of disease and risk factors, 2001: systematic analysis of population health data. Lancet 2006;367:1747-57.

20. World Health Organization. Joint NDoH-WHO Facilitators Workshop on Emergency and Essential Surgical Care towards strengthening Rural and Remote Health Facilities. 2009 (30 November 2013). http://www.who.int/surgery/education_training/ PNGreport2009.pdf

21. Ashwell HE, Barclay L. Problems measuring community health status at a local level: Papua New Guinea's health information system. Rural Remote Health 2010;10:1539.

22. World Health Organization. WHO tool for situational analysis to assess emergency and essential surgical care. 2014 (30 November 2013). http://www.wh.int/entity/surery/publications/QuickSitAnalysis EESCsurvey.pdf

23. World Health Organization. WHO Generic Essential Emergency Equipment List. 2012. http://www.who.int/surgery/publications/ EEEGenericlist_revAug2012.pdf

24. LeBrun DG, Chackungal S, Chao TE, et al. Prioritizing essential surgery and safe anesthesia for the post-2015 development agenda: operative capacities of 78 district hospitals in 7 low- and middle-income countries. Surgery 2014;155:365-73.

25. The National Research Institute, Papua New Guinea. Papua New Guinea District and Provincial Profiles. 2010 (15 July 2014). http://www. nri.org.pg/...divisions/...divisional....Web\%20Version\%20Profiles

26. Duke $T$, Wandi $F$, Jonathan $M$, et al. Improved oxygen systems for childhood pneumonia: a multihospital effectiveness study in Papua New Guinea. Lancet 2008;372;1328-33.

27. Dünser MW, Baelani I, Ganbold L. A review and analysis of intensive care medicine in the least developed countries. Crit Care Med 2006;34:1234-42.

28. Bastos PG, Knaus WA, Zimmerman JE, et al. The importance of technology for achieving superior outcomes from intensive care. Brazil APACHE III Study Group. Intensive Care Med 1996;22: $664-9$.

29. De Brouwere V, Tonglet R, Van Lerberghe W. Strategies for reducing maternal mortality in developing countries: what can we 
learn from the history of the industrialized West? Trop Med Int Health 1998;3:771-82.

30. Dubowitz G, Detlefs S, McQueen KA. Global anesthesia workforce crisis: a preliminary survey revealing shortages contributing to undesirable outcomes and unsafe practices. World J Surg 2010;34:438-44.

31. PLoS Medicine Editors. A crucial role for surgery in reaching the UN Millennium Development Goals. PLoS Med 2008;5:e182.

32. Kevau I, Watters DA. Specialist surgical training in Papua New Guinea: the outcomes after 10 years. ANZ J Surg 2006;76:937-41.

33. Ozgediz D, Jamison D, Cherian M, et al. The burden of surgical conditions and access to surgical care in low- and middle-income countries. Bull World Health Organ 2008;86:646-7.

34. Kingham TP, Kamara TB, Cherian MN, et al. Quantifying surgical capacity in Sierra Leone: a guide for improving surgical care. Arch Surg 2009;144:122-7; discussion 128

35. Cherian M, Choo S, Wilson I, et al. Building and retaining the neglected anaesthesia health workforce: is it crucial for health systems strengthening through primary health care? Bull World Health Organ 2010;88:637-9.

36. Contini S, Taqdeer A, Cherian M, et al. Emergency and essential surgical services in Afghanistan: still a missing challenge. World $J$ Surg 2010;34:473-9.

37. Kushner AL, Cherian MN, Noel L, et al. Addressing the Millennium Development Goals from a surgical perspective: essential surgery and anesthesia in 8 low- and middle-income countries. Arch Surg 2010;145:154-9.
38. Gupta S, Wong EG, Kushner AL. Scarcity of protective items against HIV and other bloodborne infections in 13 low- and middle-income countries. Trop Med Int Health 2014;19:1384-90.

39. Hendel S, Coonan T, Thomas S, et al. The rate-limiting step: the provision of safe anesthesia in low-income countries. World J Surg 2015;39:833-41.

40. Merchant A, Hendel S, Shockley R, et al. Evaluating progress in the global surgical crisis: contrasting access to emergency and essential surgery and safe anesthesia around the world. World J Surg 2015;39:2630-5.

41. Grimes CE, Henry JA, Maraka J, et al. Cost-effectiveness of surgery in low- and middle-income countries: a systematic review. World $\mathrm{J}$ Surg 2014;38:252-63.

42. Hedges JP, Mock CN, Cherian M. The political economy of emergency and essential surgery in global health. World J Surg 2010;34:2003-6.

43. Somanathan A, Morris I. Papua New Guinea (PNG) health workforce crisis: a call to action. World Bank, 2012 (6 Jun 2014). http://documents.worldbank.org/curated/en/2012/11/17196799/ papua-new-guinea-png-health-workforce-crisis-call-action

44. Hoyler M, Finlayson SR, McClain CD, et al. Shortage of doctors, shortage of data: a review of the global surgery, obstetrics, and anesthesia workforce literature. World J Surg 2014;38: 269-80.

45. Ozgediz D, Galukande M, Mabweijano J, et al. The neglect of the global surgical workforce: experience and evidence from Uganda. World J Surg 2008;32:1208-15. 